INPLASY

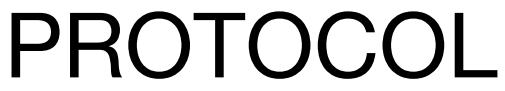

To cite: Liu et al. Efficacy and safety of moxibustion for menstrual irregularities : A protocol for systematic review and meta-analysis. Inplasy protocol 2020120042. doi: 10.37766/inplasy2020.12.0042

Received: 07 December 2020

Published: 07 December 2020

Corresponding author:

Li Liu

276816857@qq.com

Author Affiliation: Jiangxi Integrated Chinese and Western Medicine Hospital

Support: Scientific research project.

Review Stage at time of this submission: The review has not yet started.

Conflicts of interest: The authors have no conflicts of interest to disclose.

\section{Efficacy and safety of moxibustion for menstrual irregularities: A protocol for systematic review and meta-analysis}

Liu, L1; Liu, M2; Liu, M3; Gui, Y4; Sun, L5; Zuo, X6.

Review question / Objective: The purpose of this study is to evaluate the effectiveness and safety of moxibustion for treating menstrual irregularities

Condition being studied: Menstrual irregularities (MI) are one of the most common clinical gynecological diseases, with abnormal menstrual cycles, abnormal bleeding, and abdominal pain before or during menstruation as the main clinical manifestations. In modern medicine, abnormalities in the function of the pituitary gland, hypothalamus, and ovaries can affect menstruation. Currently, hormone levels in the body are mostly regulated by hormonal drugs, but these drugs can lead to hormonal imbalance, which can lead to adverse reactions. Many clinical studies have reported that moxibustion has a good effect on MI treatment, but there is no relevant systematic review. So the purpose of this study is to evaluate the effectiveness and safety of moxibustion in treating MI.

INPLASY registration number: This protocol was registered with the International Platform of Registered Systematic Review and Meta-Analysis Protocols (INPLASY) on 07 December 2020 and was last updated on 07 December 2020 (registration number INPLASY2020120042).

\section{INTRODUCTION}

Review question / Objective: The purpose of this study is to evaluate the effectiveness and safety of moxibustion for treating menstrual irregularities
Rationale: XA large number of clinical studies have reported that moxibustion has a good effect on the treatment of MI, but there is no relevant systematic review. 
Condition being studied: Menstrual irregularities (MI) are one of the most common clinical gynecological diseases, with abnormal menstrual cycles, abnormal bleeding, and abdominal pain before or during menstruation as the main clinical manifestations. In modern medicine, abnormalities in the function of the pituitary gland, hypothalamus, and ovaries can affect menstruation. Currently, hormone levels in the body are mostly regulated by hormonal drugs, but these drugs can lead to hormonal imbalance, which can lead to adverse reactions. Many clinical studies have reported that moxibustion has a good effect on MI treatment, but there is no relevant systematic review. So the purpose of this study is to evaluate the effectiveness and safety of moxibustion in treating MI.

\section{METHODS}

Search strategy: The following 8 electronic databases will be searched, including PubMed, Embase, the Cochrane Library, China National Knowledge Infrastructure (CNKI), Web of Science, Chinese Scientific Journal Database(VIP), Wanfang Database, and Chinese Biomedical Literatures Database (CBM) from their inception to 1 December 2020 without any restrictions.

Participant or population: The patients who are diagnosed with $\mathrm{MI}$ regardless of ethnic group, severity, syndrome type and source of cases clinically will be included. Studies that MI combined with other basic diseases will be excluded.

Intervention: The studies that used various forms of moxibustion (e.g. direct or indirect moxibustion, heat-sensitive moxibustion, warm needling moxibustion, or saltseparated moxibustion, etc.) as the single therapy or as the main part of a combination treatment with other interventions (e.g. western medicine, etc) will be considered. And the studies that moxibustion was used as an ancillary treatment will be excluded.
Comparator: The control interventions will include: positive interventions (e.g. western medicine), no intervention, placebo or sham moxibustion. The choice of specific forms, as follows:(1). Moxibustion VS positive interventions;(2). Moxibustion+ positive interventions VS positive interventions.

Study designs to be included: All relevant randomised controlled trials (RCTs) or quasi-RCTs will be included.

Eligibility criteria: All the RCTs or quasiRCTs of moxibustion for patients with MI will be included without publication status restriction, review articles, case reports, conference abstracts, cross-sectional studies, and all observational studies will be excluded.

Information sources: We will electronically search the randomized controlled trials in the following databases: including the PubMed, Embase, the Cochrane Library, China National Knowledge Infrastructure (CNKI), Web of Science, Chinese Scientific Journal Database(VIP), Wanfang Database and Chinese Biomedical Literatures Database (CBM) from their inception to 1 December 2020 without any restrictions. The search strategy that will be run in the PubMed and adjusted to fit the other database when necessary.

\section{Main outcome(s): The effective rate}

Additional outcome(s): Incidence of any adverse events.

Quality assessment / Risk of bias analysis: Based on the Cochrane Handbook for Systematic Reviews of Interventions, the risk of bias in all studies will be assessed by 2 authors independently using the Cochrane risk of a bias assessment tool. Six areas of each trial will be evaluated: generation of random sequences, allocation concealment, blinding method, incomplete outcome data, selective reporting and other bias. Each domain will be divided into three levels of bias: unclear risk, high risk and low risk and "Risk of bias" will be filled in. And any differences 
will be resolved through negotiation or consulting with other reviewers.

Strategy of data synthesis: We will use RevMan 5.3.0 software to perform the meta-analysis. We will summarize data using risk ratios (RR) with $95 \% \mathrm{Cl}$ for binary outcomes or mean difference (MD) with $95 \% \mathrm{Cl}$ for continuous outcomes. $\mathrm{I}^{2}$ value and $P$-value will be used to test the degree of heterogeneity. When $\mathbf{P}>0.1, \mathrm{I}^{2}<50 \%$, no heterogeneity was considered between the studies and the fixed effect model will be used for statistical analysis; otherwise, the random effect model will be used. If there was significant clinical heterogeneity between studies, only descriptive analysis was performed. For a study with incomplete result data, we will try to contact the first author.

Subgroup analysis: In order to explore the possible causes of heterogeneity, we will conduct subgroup analysis if there are a sufficient number of studies (at least 10 trials). In addition, if we do not observe the predicted effect in all the subjects, the subgroup analysis can help us find out whether the treatment is effective in some subgroups.

Sensibility analysis: In order to assure the robustness of our results, We will conduct sensitivity analysis to eliminate the impact of low-quality studies, with the premise of significant heterogeneity still exists right after validation of inputted data and subgroup analysis. We will contrast the results of these two meta-analyses and decide whether to exclude low-quality researches based on impact on the pooled effect size, sample size and strength of evidence. Nevertheless, if all included studies are at high risk of bias, we will not conduct sensitivity analysis.

Language: English.

Country(ies) involved: China.

Keywords: Menstrual irregularities, moxibustion, randomized controlled trial, protocol.
Contributions of each author:

Author 1 - Li Liu - drafted the manuscript.

Email: 280433108@qq.com

Author 2 - Min Liu - provided statistical expertise.

Email: 276816857@qq.com

Author 3 - Meinian Liu - contributed to the development of the selection criteria.

Email: 1569600832@qq.com

Author 4 - Yufang Gui - contributed to the development of the selection criteria.

Email: 1569600832@qq.com

Author 5 - Lei Sun - Provided feedback and approved the final manuscript.

Email: 123687223@qq.com

Author 6 - Xiaoyun Zuo - use the software to analyze the data and write the original draft.

Email: 5682346@qq.com 